\title{
Human Skin Based Triboelectric Nanogenerators for Harvesting Biomechanical Energy and as Self- Powered Active Tactile Sensor System
}

\author{
Ya Yang, ${ }^{\dagger, \perp}$ Hulin Zhang, ${ }^{t, \neq, \perp}$ Zong-Hong Lin, ${ }^{\dagger}$ Yu Sheng Zhou, ${ }^{\dagger}$ Qingshen Jing, ${ }^{\dagger}$ Yuanjie Su, ${ }^{\dagger}$ Jin Yang, ${ }^{\dagger}$ \\ Jun Chen, ${ }^{\dagger}$ Chenguo Hu, ${ }^{\ddagger}$ and Zhong Lin Wang ${ }^{\dagger, \S, *}$ \\ †School of Materials Science and Engineering, Georgia Institute of Technology, Atlanta, Georgia 30332-0245, United States, ${ }^{\ddagger}$ Department of Applied Physics, \\ Chongqing University, Chongqing 400044, China, and ${ }^{\S}$ Beijing Institute of Nanoenergy and Nanosystems, Chinese Academy of Sciences, China. ${ }^{\perp}{ }^{\text {Ya }}$ Yang and Hulin \\ Zhang contributed equally to the work.
}

\begin{abstract}
We report human skin based triboelectric nanogenerators (TENG) that can either harvest biomechanical energy or be utilized as a self-powered tactile sensor system for touch pad technology. We constructed a TENG utilizing the contact/ separation between an area of human skin and a polydimethylsiloxane (PDMS) film with a surface of micropyramid structures, which was attached to an ITO electrode that was grounded across a loading resistor. The fabricated TENG delivers an opencircuit voltage up to $-1000 \mathrm{~V}$, a short-circuit current density of $8 \mathrm{~mA} / \mathrm{m}^{2}$, and a power density of $500 \mathrm{~mW} / \mathrm{m}^{2}$ on a load of $100 \mathrm{M} \Omega$, which can be used to directly drive tens of green light-emitting diodes. The working mechanism of the TENG is based on the charge transfer between the ITO electrode and ground via modulating the separation distance between the tribo-charged skin patch and PDMS film. Furthermore, the TENG
\end{abstract}
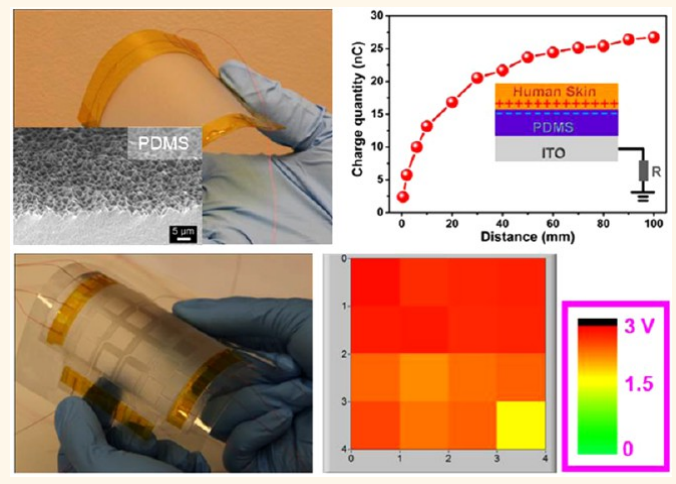
has been used in designing an independently addressed matrix for tracking the location and pressure of human touch. The fabricated matrix has demonstrated its self-powered and high-resolution tactile sensing capabilities by recording the output voltage signals as a mapping figure, where the detection sensitivity of the pressure is about $0.29 \pm 0.02 \mathrm{~V} / \mathrm{kPa}$ and each pixel can have a size of $3 \mathrm{~mm} \times 3 \mathrm{~mm}$. The TENGs may have potential applications in human-machine interfacing, micro/nano-electromechanical systems, and touch pad technology.

KEYWORDS: triboelectric nanogenerator $\cdot$ human skin $\cdot$ self-powered $\cdot$ tactile sensor $\cdot$ power density $\cdot$ touch pad

$\Gamma$ arvesting biomechanical energy from human motion has attracted increasing attention in the past decade for potential applications in charging portable electronic device batteries and selfpowered sensor systems. ${ }^{1-3}$ Renewable biomechanical energy harvesting techniques based on electromagnetic, electrostatic, and piezoelectric mechanisms have been developed to address these problems. ${ }^{4-7}$ Recently, the triboelectric effect has been utilized to scavenge mechanical energy from irregular vibrations, sliding, and rotations, leading to the invention of triboelectric nanogenerators (TENGs). ${ }^{8-11}$ Based on the triboelectric effect coupled with electrostatic induction, TENGs have been used to harvest biomechanical energy from human-driven shaking and squeezing. ${ }^{12-14}$ All the previously reported biomechanical energy harvesting techniques are based on external devices attached to the human body. An interesting question is what if the human body itself can be used as a power-generating material so that energy can be generated in the touching process. According to the triboelectric series, ${ }^{15}$ human skin as one of the triboelectric materials can be used to fabricate this kind of device by utilizing TENG techniques. However, TENGs usually need metal electrodes deposited on the triboelectric materials for effective electrical output, which cannot be realized on human skin. Developing single-electrode-based TENGs can solve this problem and enable us to harvest
*Address correspondence to zlwang@gatech.edu.

Received for review July 24, 2013 and accepted August 31, 2013.

Published online September 05, 2013 $10.1021 / \mathrm{nn} 403838 \mathrm{y}$

(C) 2013 American Chemical Society 
biomechanical energy from human skin as well as building new touch pad technology.

Usually, a tactile sensor refers to a transducer that is sensitive to touch, force, or pressure. ${ }^{16}$ This type of sensor has a wide variety of applications for robotics, human-machine interfacing, and security systems. Based on the piezoresistive effect, an organic transistor array, as an artificial electronic skin, was successful in the tactile sensing of human fingers. ${ }^{17-19}$ Moreover, the piezotronic effect has been used to fabricate a twoterminal transistor array matrix for detecting strain distribution with a density of 8464 devices per $1 \mathrm{~cm}$ square. ${ }^{20}$ An external power source is required to drive these tactile sensors. Self-powered nanotechnology utilizes energy harvesters instead of external power sources or conventional batteries to drive the sensors. $^{21,22}$ TENGs have been utilized as self-powered sensors for sensing mercury ions, catechin, and change of magnetic field. ${ }^{23-25}$

In this paper, we present a human skin based TENG that is based on periodic contact/separation between a human skin patch and a polydimethylsiloxane (PDMS) film with a surface of micropyramid structures on an ITO electrode. Due to the coupling between the triboelectric effect and the electrostatic effect, the periodic change of distance between two surfaces results in charge transfer between the ITO electrode and the ground, thus driving the flow of electrons across an external load. The fabricated TENG can produce an open-circuit voltage up to $-1000 \mathrm{~V}$ and a short-circuit current density of $8 \mathrm{~mA} / \mathrm{m}^{2}$, which can be used to drive tens of green light-emitting diodes (LEDs) instantaneously. Moreover, a matrix of the independently addressable TENGs has been utilized in self-powered and high-resolution tactile sensing by recording the output voltage signals as a mapping figure. The touched location and pressure can be determined by analysis of the mapping figures. This work is important progress toward the practical applications of skinbased biomechanical energy harvesting techniques and self-powered sensor systems.

\section{RESULTS AND DISCUSSION}

The fabricated TENG consists of a human skin patch and a PDMS film on an ITO electrode. Figure 1a illustrates a photograph of the PDMS film, where the microstructured surface was fabricated using a $\mathrm{Si}$ micropyramid template. Figure $1 \mathrm{~b}$ shows a scanning electron microscope (SEM) image of the fabricated PDMS film. The surface is uniformly covered by micropyramid structures with sizes less than $5 \mu \mathrm{m}$. The micropyramid structures were further confirmed by the cross-sectional SEM image of the PDMS film, as shown in Figure 1c. The corresponding thickness of the flexible PDMS film is about $400 \mu \mathrm{m}$, as shown in Figure S1. The micropyramid structure of the PDMS film can be used to induce a larger triboelectric charge density to enhance the output performance of TENG. Additionally this micropyramid structure can also change some other physical properties such as the wettability of the polymer surface. Figure $1 \mathrm{~d}$ shows that the contact angle between the PDMS polymer of the microstructured surface and water $\left(131^{\circ}\right)$ is obviously larger than that $\left(113^{\circ}\right)$ of the PDMS polymer without microstructure, as illustrated in Figure S2. The increase of the contact angles is beneficial since water droplets can easily roll off the surfaces of the device to take with them dirt and contaminants, realizing a selfcleaning function. ${ }^{26}$

Figure $1 \mathrm{~d}$ shows the measured open-circuit voltages of the fabricated TENG by utilizing an electrometer with very large input resistance, where the voltage can reach $-1000 \mathrm{~V}$ during fast contact/separation between the skin and PDMS film. The obtained negative voltages are associated with the negative triboelectric charges on the surface of the PDMS film. The corresponding short-circuit current density of the device reached $8 \mathrm{~mA} / \mathrm{m}^{2}$, as shown in Figure 1e. Figure $1 \mathrm{~g}$ and $\mathrm{h}$ present the corresponding output voltage and the current density when a load resistor of $100 \mathrm{M} \Omega$ was connected between the ITO electrode and ground, respectively. The device produced an output voltage peak of $180 \mathrm{~V}$ with a current density peak of $2.8 \mathrm{~mA} / \mathrm{m}^{2}$, resulting in an output power density of $500 \mathrm{~mW} / \mathrm{m}^{2}$. The obtained output performance of the device with a micropyramid-structured surface is much larger than that without the microstructures, as illustrated in Figures S3 and S4. To demonstrate that the produced energy can be used as an effective power source, the PDMS film on the ITO electrode was attached to the back of a human hand, which was then connected to tens of green LEDs. Upon a fast swing of the hand, these green LEDs can be lighted up by the produced energy, as shown in movie file-1 (see the Supporting Information).

The working principle of the fabricated TENG is schematically depicted in Figure 2 by the coupling of contact electrification and electrostatic induction. In the original position, the surfaces of the skin and PDMS fully contact each other, resulting in charge transfer between them. According to the triboelectric series, ${ }^{15}$ electrons were injected from the skin to the PDMS since PDMS is more triboelectrically negative than skin, which is the contact electrification process. The produced triboelectric charges with opposite polarities are fully balanced/screened, leading to no electron flow in the external circuit, as shown in Figure 2a. Once a relative separation between PDMS and skin occurs, these triboelectric charges cannot be compensated. The negative charges on the surface of the PDMS can induce positive charges on the ITO electrode, driving free electrons to flow from the ITO electrode to the ground, as depicted in Figure $2 \mathrm{~b}$. This electrostatic induction process can give an output voltage/current 

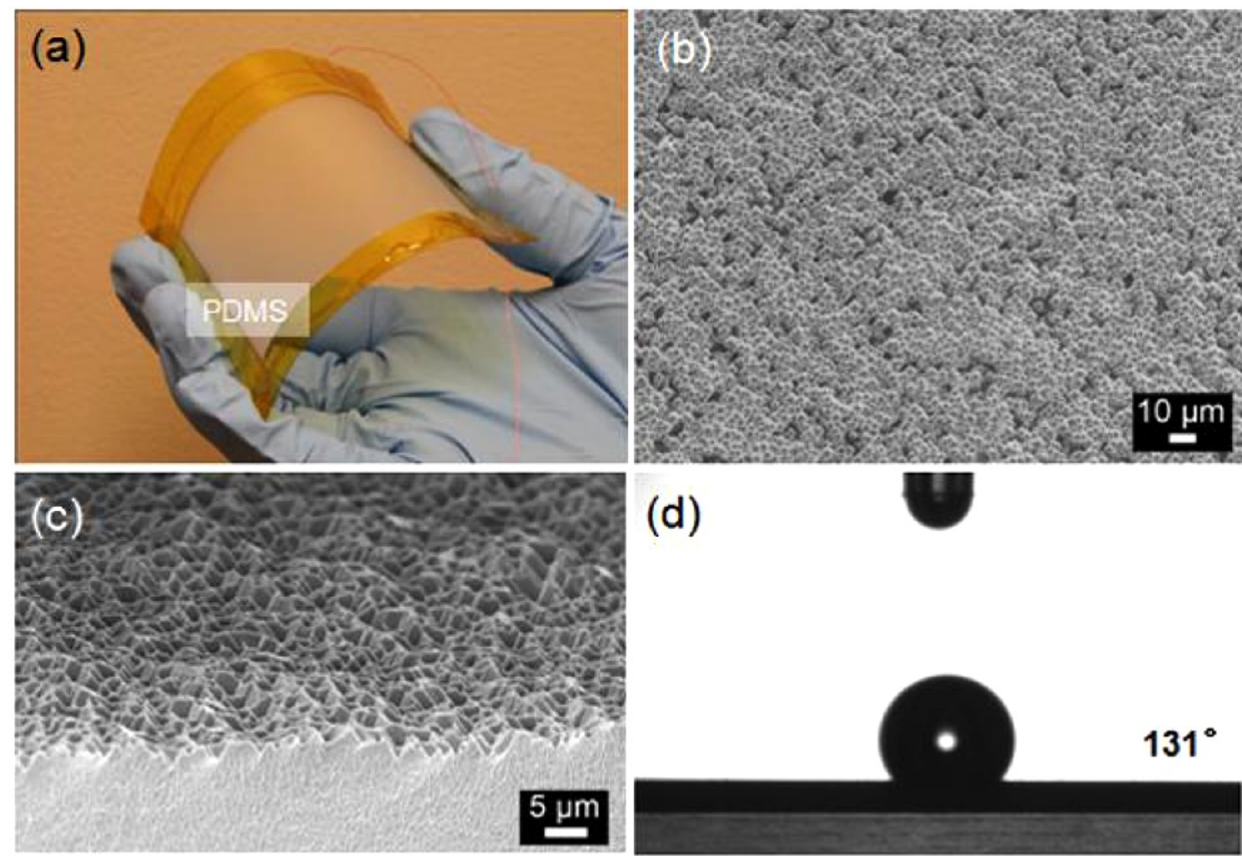

(d)
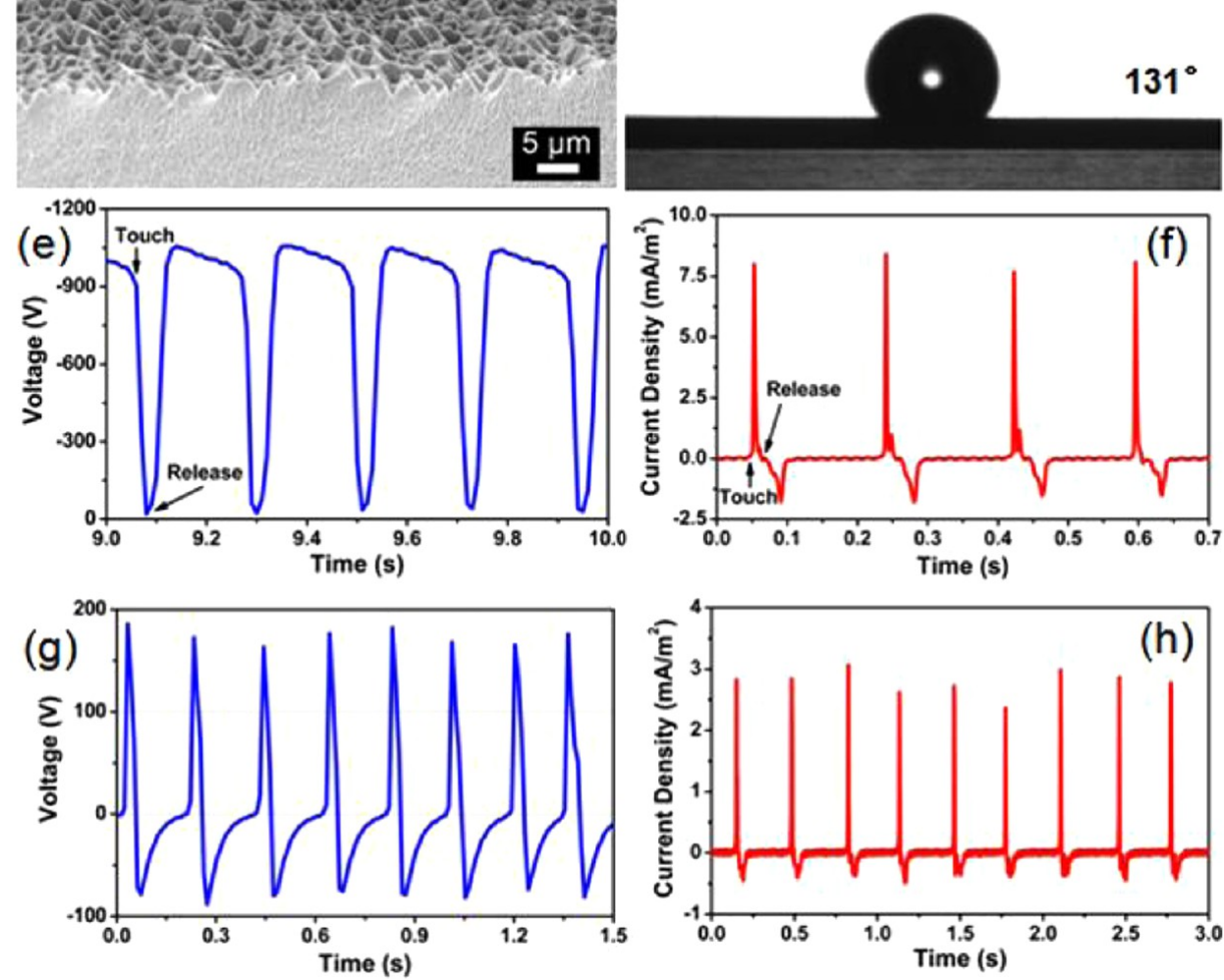

Figure 1. (a) Photograph of the fabricated PDMS film on the ITO electrode. (b) SEM image of the PDMS surface with micropyramid structures. (c) Tilted SEM image of the PDMS surface. (d) Photograph of the contact angle between the PDMS and water. (e) Measured open-circuit voltage of the device up to $-1000 \mathrm{~V}$. (f) Measured short-circuit current density of the TENG. $(g, h)$ Measured output voltage $(g)$ and current density $(h)$ of the TENG with the used loading resistance of $100 \mathrm{M} \Omega$.

signal. When negative triboelectric charges on the PDMS are fully screened from the induced positive charges on the ITO electrode by increasing the separation distance between the PDMS and skin, no output signals can be observed, as illustrated in Figure 2c. Moreover, when the skin was reverted to approach the PDMS, the induced positive charges on the ITO electrode decrease and the electrons flow from the ground to the ITO electrode until the skin and PDMS fully contact each other again, resulting in a reversed output voltage/current signal, as shown in Figure $2 \mathrm{~d}$. This is a full cycle of the electricity generation process for the TENG.
To obtain a more quantitative understanding of the proposed working principle of the TENG, we employed finite element simulations to calculate the electric potential distribution in the TENG and the charge transfer between the ITO electrode and ground using COMSOL. The constructed model is based on a skin patch and a PDMS film on the ITO electrode with the dimensions $45 \mathrm{~mm} \times 65 \mathrm{~mm}$ and thickness of $1 \mathrm{~mm}$ for skin, $0.5 \mathrm{~mm}$ for PDMS film, and $1 \mathrm{~mm}$ for ITO electrode, as depicted in Figure 3a. The ITO electrode was connected with the ground. The triboelectric charge densities on the skin and PDMS film were assumed to 
be +10 and $-10 \mu \mathrm{C} / \mathrm{m}^{2}$, respectively. Figure $3 \mathrm{~b}$ shows the calculated results of the electric potential distribution in the TENG under the different separation

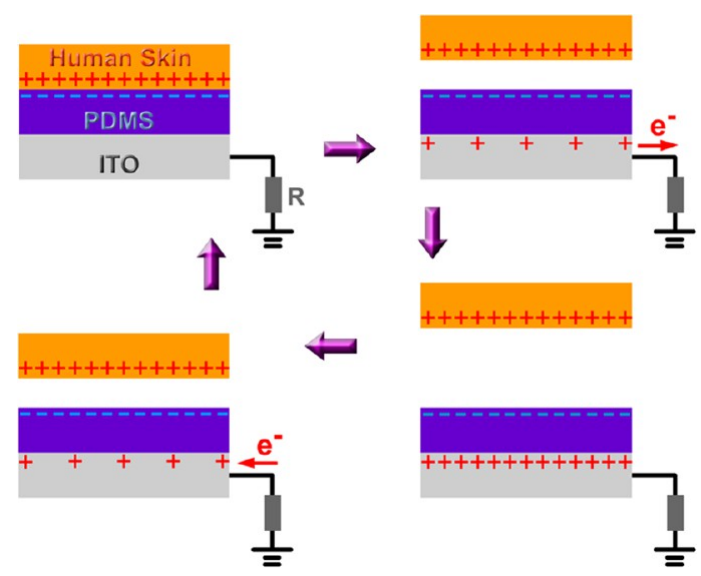

Figure 2. Electricity generation process in a full cycle. (a) Full contact between human skin and the PDMS film. (b) The two surfaces are separated by a small distance. (c) The two surfaces are separated by a large distance. (d) The separated surfaces are approaching each other. distances of $0.5,10,30$, and $60 \mathrm{~mm}$, respectively. When the skin and PDMS fully contact with each other, the electric potentials on both the skin and PDMS approach zero. The electric potential difference was found to increase dramatically with increasing the separation distance. When they are separated by $60 \mathrm{~mm}$, the electric potential on the skin surface reaches $1.2 \times 10^{4} \mathrm{~V}$, while the electric potential on the PDMS is still close to zero, which is associated with contact with the ITO electrode/ground. As illustrated in Figure $3 c$, the amount of total charges on the ITO electrode was plotted as a function of separation distance between the skin and PDMS. It increases with increasing the separation distance, revealing that charge transfer decreases with increasing distance. These results are consistent with the measured change in charge quantity when the contacted skin was removed from the PDMS film, as shown in Figure $3 \mathrm{~d}$.

To demonstrate the capability of the TENG to scavenge the biomechanical energy in daily life, we fabricated the TENG on a commercial cell phone,
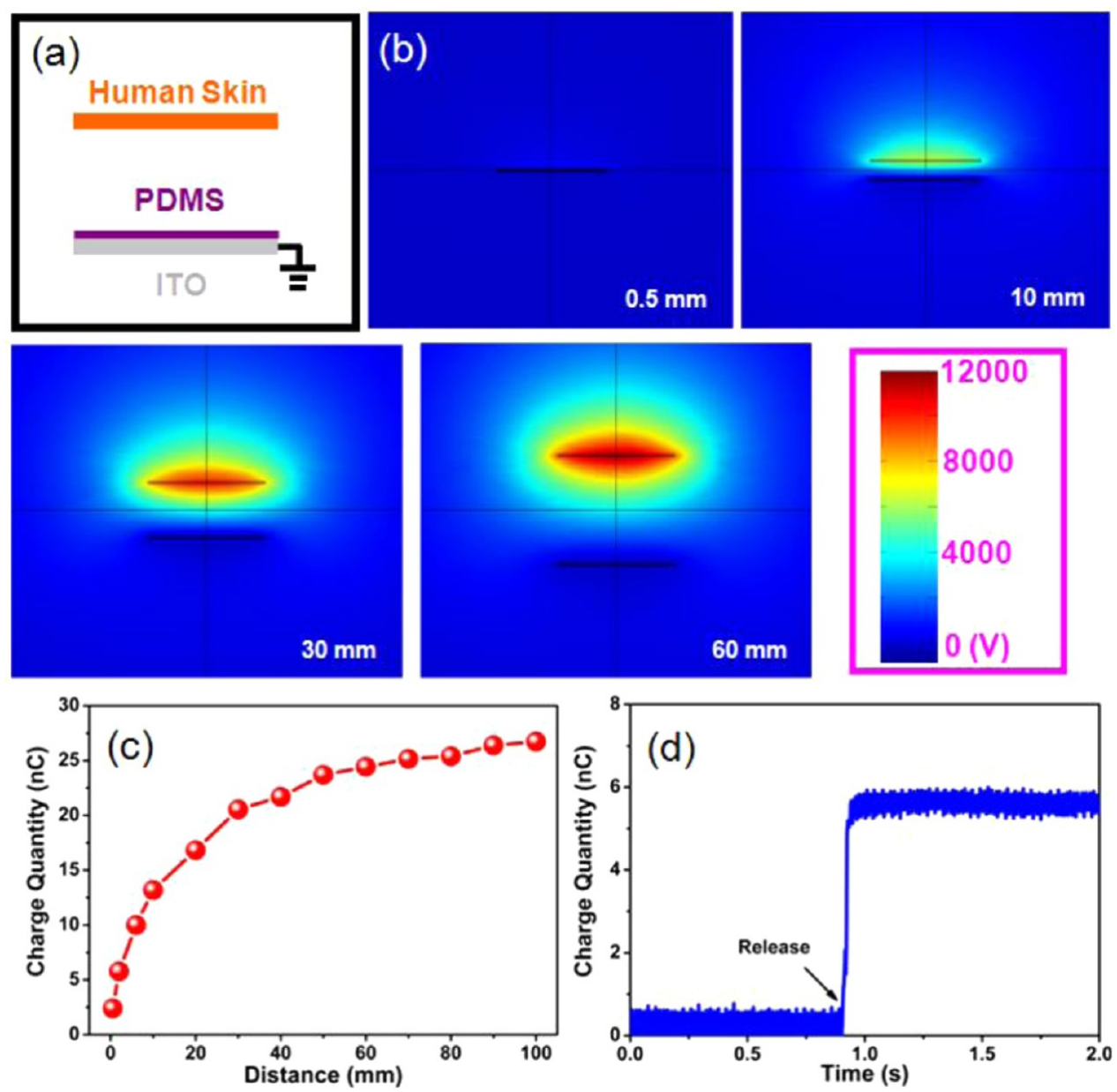

Figure 3. (a) Schematic diagram of the model for the calculation. (b) Finite element simulation of the potential distribution in the TENG for the different separation distances between the skin and PDMS film. (c) The total charges on the ITO electrode as a function of the separation distance between the skin and PDMS film. The increase in total charges is a result of the electron flow from ITO to the ground. (d) Measured charge quantity when the applied pressure on the PDMS film was released. 

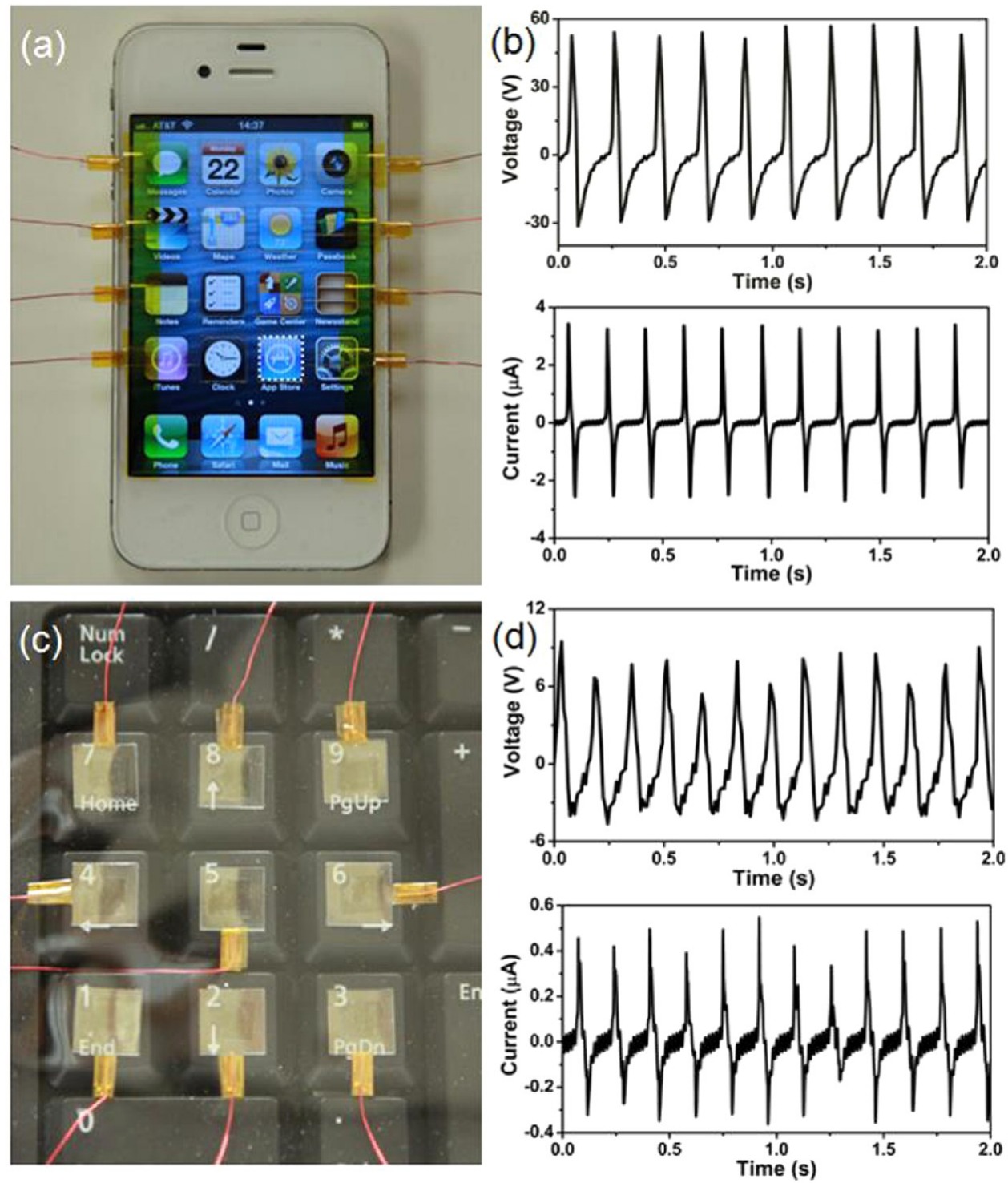

Figure 4. (a) Photograph of the PDMS film without the micropyramid structures and the ITO electrodes attached on a commercial cell phone. (b) Measured output voltage and current of the TENG (a) upon the touch of a human hand with the used loading resistance of $100 \mathrm{M} \Omega$. (c) Photograph of the PDMS film without the micropyramid structures and the ITO electrodes attached on a commercial keyboard. (d) Measured output voltage and current of the TENG (c) upon the touch of a human finger with the used loading resistance of $100 \mathrm{M} \Omega$.

where each ITO electrode has a size of about $1 \mathrm{~cm} \times$ $1 \mathrm{~cm}$, and the PDMS film without the micropyramid structures was then overlaid on the ITO electrode surface, as shown in Figure $4 a$. Figure $4 b$ presents the obtained output voltage of about $60 \mathrm{~V}$ with the corresponding output current of about $3.5 \mu \mathrm{A}$ when the screen of the TENG-based cell phone was touched by a human hand, resulting in an output power of about $0.2 \mathrm{~mW}$. The designed TENG-based cell phone can have both the functionality of the commercial cell phone and the biomechanical energy harvesting ability, as shown in movie file-2 (see the Supporting Information). In addition, this demonstration shows the potential of adding another sensor system on top of the existing sensor network for detecting different signals.
Another demonstration is where the TENG was attached to a commercial keyboard, as displayed in Figure $4 c$. When each key was touched by a human finger, the TENG can produce an output voltage of about $8 \mathrm{~V}$, an output current of about $0.5 \mu \mathrm{A}$, and an output power of $4 \mu \mathrm{W}$ on a load of $100 \mathrm{M} \Omega$. This TENG technique can be applied to all commercial keyboards without affecting the functionality of the devices for harvesting wasted typing-induced biomechanical energy, as illustrated in movie file-3 (see the Supporting Information).

To illustrate the potential applications of the TENG, we demonstrated that the TENG can be utilized as a self-powered tactile sensor for monitoring the local touching actions of human fingers or hands. Figure 5 a displays a photograph of a $4 \times 4$ matrix of 

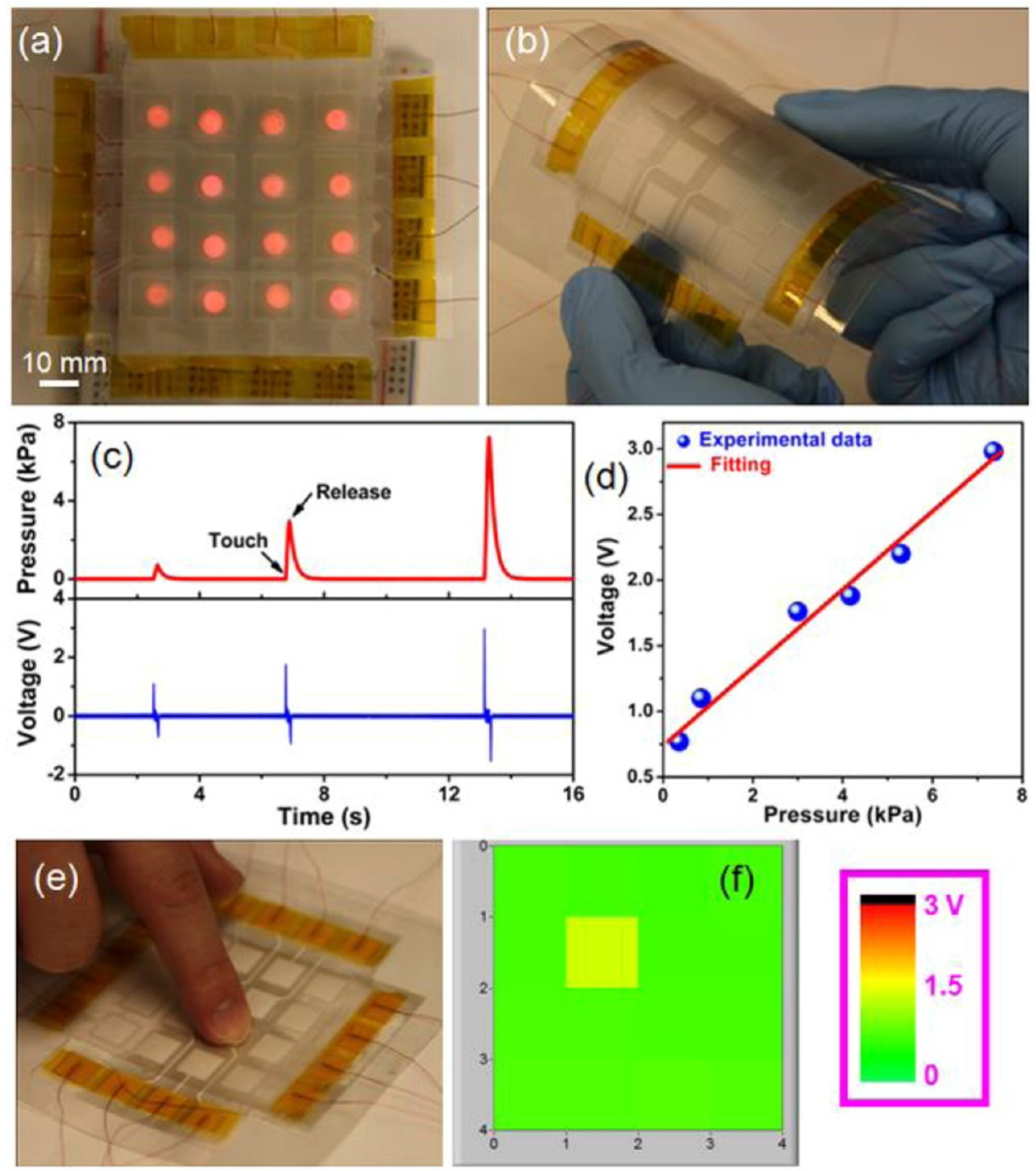

Figure 5. (a) Photograph of the device on the red LED array, exhibiting the transparent feature. (b) Photograph of the bent device. (c) Output voltages of one device in the matrix under different pressures. (d) Output voltage as a function of the applied pressure. The red line corresponds to the linear fitting function. (e) Photograph of the matrix touched by a human finger. (f) Measured positive output voltage mapping figure for the touching in $e$.

the fabricated ITO electrode array, where each electrode with a size of $1 \mathrm{~cm} \times 1 \mathrm{~cm}$ was connected with a load resistor of $10 \mathrm{M} \Omega$ and then with a ground. The fabricated matrix was completely covered by a PDMS film as the sensitive unit to be touched. The device exhibits both transparent and flexible features, as illustrated in Figure $5 a$ and $b$, respectively. When the PDMS film was touched by a human finger with a representative pressure of less than $10 \mathrm{kPa}^{27}$ the corresponding output voltage signals were observed, as illustrated in Figure $5 \mathrm{c}$. The magnitude of the output voltage is proportional to the applied pressures in the experiments, where the corresponding pressure was measured by a commercial pressure sensor based on the piezoresistive effect. When a pressure of about $7.3 \mathrm{kPa}$ induced by a finger was applied on the PDMS film, a positive output voltage peak of about $3 \mathrm{~V}$ can be observed, as displayed in Figure S5. When the applied pressure on the PDMS was released, a negative voltage peak appears. As compared with the data in Figure 1g, the smaller output voltage is associated with a smaller loading resistance. The reset time (defined as the time needed to recover to the original value before applying pressure) of the tensile senor is about $0.1 \mathrm{~s}$, which is much smaller than that $(0.7 \mathrm{~s})$ of the commercial pressure sensor. The detection limit of the pressure is about $0.4 \mathrm{kPa}$, producing an output voltage of $0.77 \mathrm{~V}$, as depicted in Figure S6. As shown in Figure 4d, the output voltage of the fabricated sensor exhibits a linear relationship with the applied pressure by fitting the data in Figures 5c, S6, and S7, where the detection sensitivity of the pressure is about $0.29 \pm 0.02 \mathrm{~V} / \mathrm{kPa}$. The increase of the output voltage with an increase in pressure is due to the increase of the effective contact area between the two triboelectric materials, resulting in a larger amount of triboelectric charges. ${ }^{14}$ 

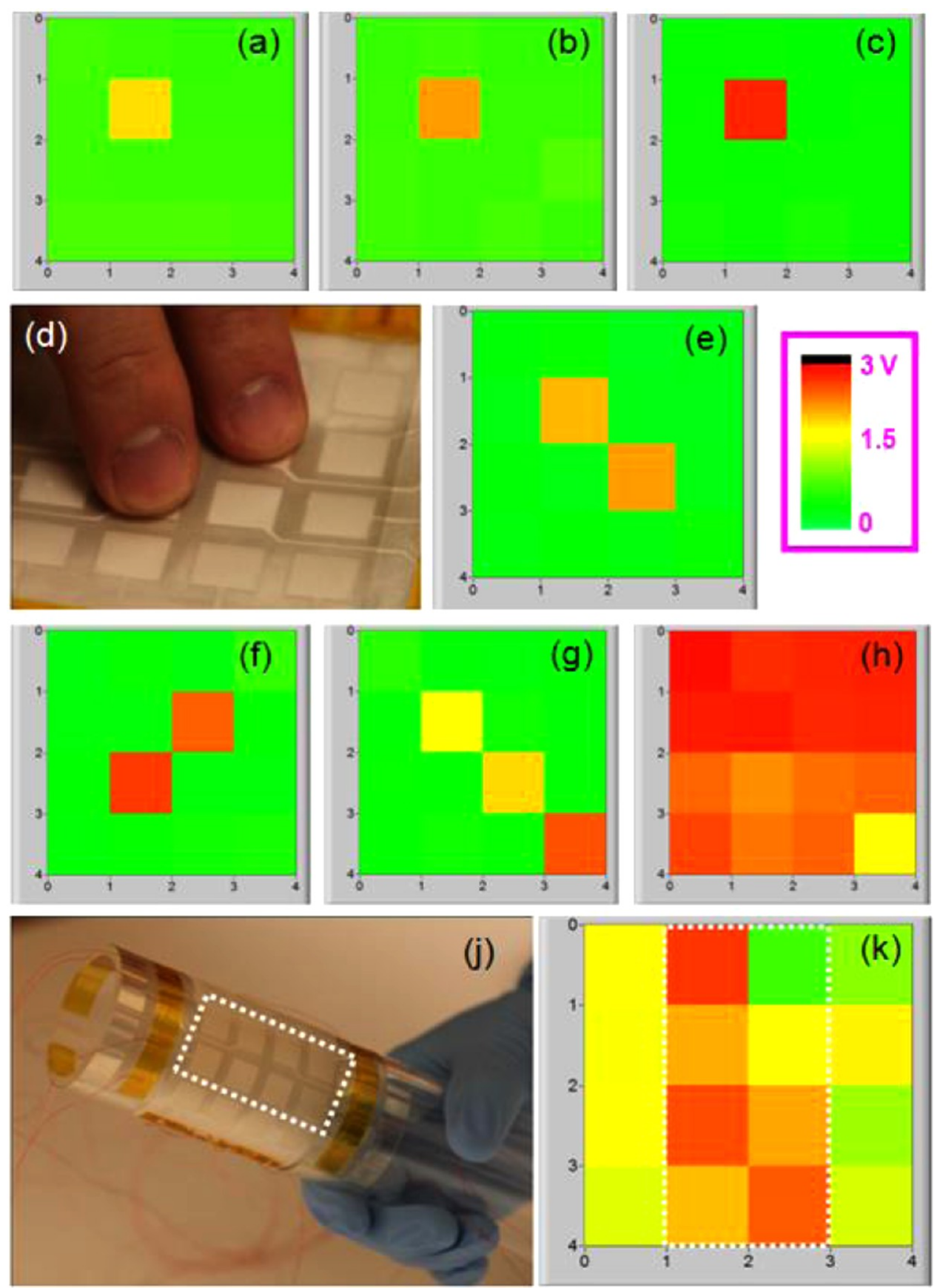

Figure 6. $(a-c)$ Measured mapping figures under the different pressures. (d) Photograph of the matrix touched by two human fingers. (e) Measured mapping figure when the two devices in the matrix were touched in (d). (f-h) Measured mapping figures when two (f), three (g), and all devices (h) in the matrix were touched. (j) Photograph of the fabricated matrix attached to an acrylic tube. (k) Measured mapping figure when the device in (j) was touched by a human hand.

The output voltage signals of 16 devices were recorded in real time as a mapping figure. By addressing and monitoring the positive output voltage signals in 16 channels of the tactile sensor system, the touch information of a human finger including the position and pressure can be attained by analysis of the measured mapping figures. As displayed in Figure 5e, when the sixth device in the matrix was touched, a positive voltage signal of $1.4 \mathrm{~V}$ was observed in the obtained mapping figure, as shown in Figure $5 \mathrm{f}$.
According to the data in Figure $5 d$, the applied pressure on the device can be calculated to be about $2.2 \mathrm{kPa}$. If a larger pressure is applied on the device, larger output voltage signals can be observed in the mapping figures (Figure $6 a-c)$. Figure $6 d$ presents a photograph of the device when the 6th and 11th devices in the matrix were simultaneously touched. Two almost equal pressures of about $4.9 \mathrm{kPa}$ can be confirmed by the analysis of the same obtained output voltage signals in the corresponding mapping image, 

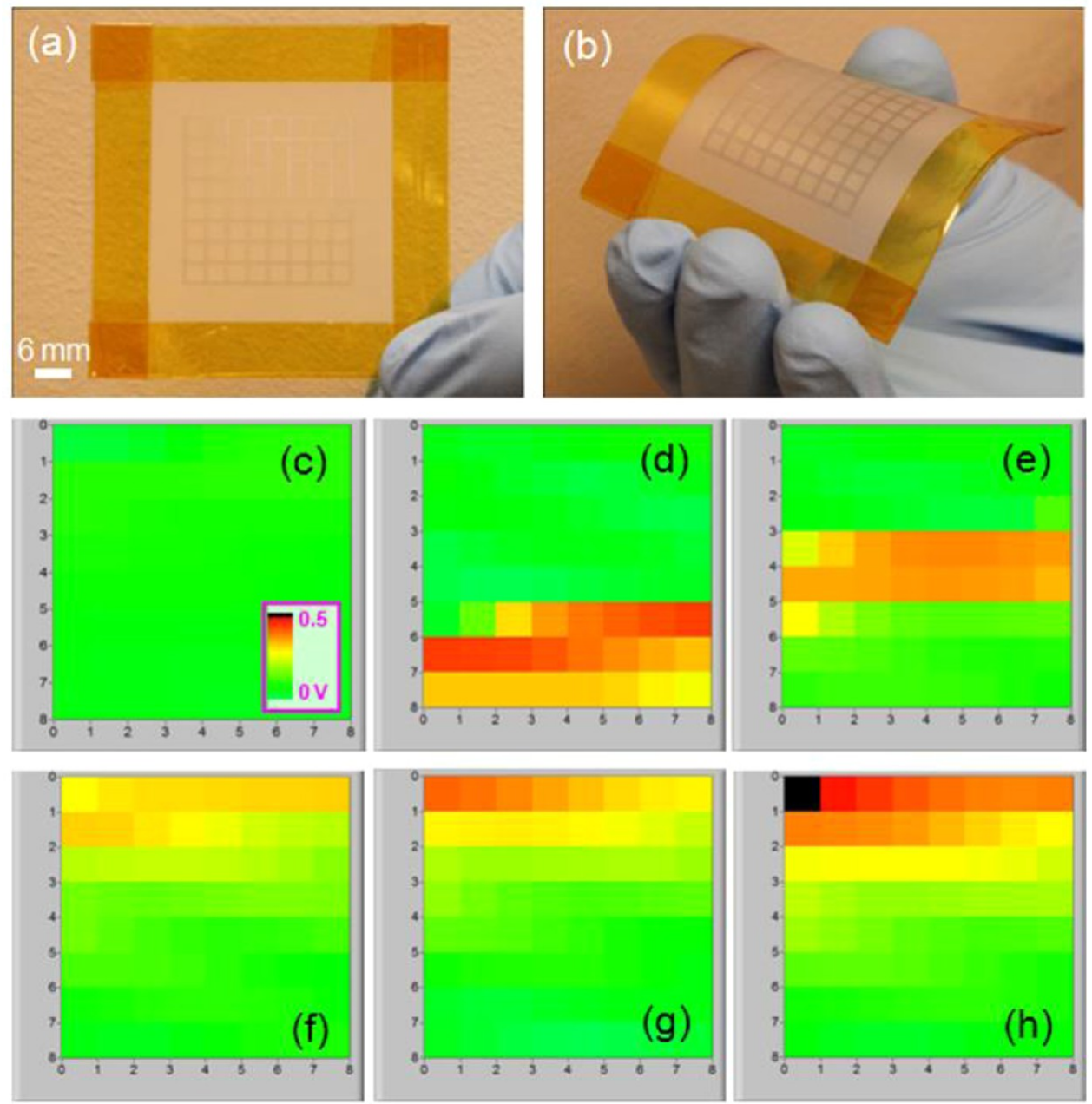

Figure 7. (a) Photograph of the fabricated $8 \times 8$ matrix with each pixel being $3 \mathrm{~mm} \times 3 \mathrm{~mm}$. (b) Photograph of the bent sensor system. (c) Mapping figure of the sensor system without touching, showing that no output voltage signals can be observed. (d-f) Measured mapping figures when the bottom (d), middle (e), and top (f) areas were touched by the side surface of a human hand. $(g, h)$ Measured mapping figures when the applied forces were increased at the top area of the matrix.

as shown in Figure 6e. Figure $6 \mathrm{f}$ displays the response of the device to the localized pressure induced by two fingers when the matrix was rotated by 90 degrees. The obtained mapping figure clearly reveals that the 7th and 10th devices were touched, where the produced pressures of about $7.0 \mathrm{kPa}$ are larger than those in Figure 6e. When the pressures were applied along the diagonal line of the matrix by using the side surface of the human hand, distinctive changes in the output voltage signals can be observed, revealing an increase of the pressure from 3.2 to $6.0 \mathrm{kPa}$ along the diagonal line, as illustrated in Figure $6 \mathrm{~g}$. When the pressures were applied on all 16 devices by using the human hand, the recorded mapping figure (Figure $6 \mathrm{~h}$ ) shows that all the devices are functional and the pressures on the 1st-8th devices are obviously larger than those on the 9th-16th devices. The real-time detection of the touching actions on the flexible devices is a desirable feature for sensors embedded in robots or prosthetic devices. Figure $6 \mathrm{j}$ shows a photograph of the flexible matrix attached to a transparent acrylic tube. When the pressures were applied on the tube surface by using the human hand, the largest pressure of about $7.3 \mathrm{kPa}$ occurs at the marked area (the white dashed line in Figure 6j), which is consistent with the measured mapping figure, as shown in Figure 6k.

The resolution of the present device is limited by the size of the ITO electrode unit on the polyester substrate. To enhance the detection resolution, an $8 \times 8$ matrix with a pixel size of $3 \mathrm{~mm} \times 3 \mathrm{~mm}$ was fabricated, where the PDMS film covered the surface of the polyester substrate. Figure 7a shows a photograph of the matrix, where 64 pixels were created in the total area of $3 \mathrm{~cm} \times 3 \mathrm{~cm}$ and the fabricated device exhibits a flexible character, as illustrated in Figure $7 \mathrm{~b}$. By monitoring the positive output voltage of each independently functioning pixel in the matrix, a spatial profile of the touching actions can be readily shown by addressing all the pixels. The output voltage mapping test was then performed on the self-powered sensor system without applying pressure, demonstrating 
that no output signals were observed, as shown in Figure 7c. To demonstrate the tactile sensing capability of the sensor system, the bottom, middle, and top areas of the matrix were touched by utilizing the side surface of the human hand, as shown in Figure $7 d-f$, respectively. The recorded output voltage signals appear in the corresponding areas, indicating that all of the devices on the 64 pixels are functional. As depicted in Figure $7 f-h$, presenting the difference of the measured mapping figures under different pressures, the output voltage signals increase with increasing the pressure, which is consistent with the results in Figure $5 \mathrm{e}-\mathrm{h}$. In this study, we have demonstrated the methodology of fabricating a flexible and transparent matrix for self-powered touch pad technology. Miniaturization of the device for a higher resolution is possible by utilizing other techniques to obtain a micro/nanoscale electrode array. As compared with the sensors used for touch pads today, the advantages of this technology include the simple structure, low cost, and self-powered capability.

\section{CONCLUSION}

In summary, we have demonstrated a human skin based TENG, consisting of a skin patch, a PDMS film with a micropyramid-structured surface, and an ITO electrode connected with a ground across an external load. The mechanism of TENG is based on the charge transfer between the ITO electrode and the ground by utilizing the contact/separation between the skin patch and the PDMS film. By converting the biomechanical energy into electricity, the TENG produces an open-circuit voltage up to $-1000 \mathrm{~V}$, a short-circuit current density of $8 \mathrm{~mA} / \mathrm{m}^{2}$, and a power density of $500 \mathrm{~mW} / \mathrm{m}^{2}$ on a load resistance of $100 \mathrm{M} \Omega$, which can be used to directly light up tens of green LEDs. Moreover, the fabricated TENGs have been used to design an independently addressable matrix for monitoring the localized touching actions of human skin. The matrix exhibits both transparent and flexible characteristics, where each pixel can have a size of $3 \mathrm{~mm} \times$ $3 \mathrm{~mm}$. The tactile information including the position and pressure can be determined by analysis of the realtime recording of positive output voltage signals in the matrix as a mapping figure. The detection sensitivity of the pressure is about $0.29 \pm 0.02 \mathrm{~V} / \mathrm{kPa}$. The size of each sensor unit can be significantly reduced so that the spatial resolution can be largely improved. This work pushes forward a significant step toward the practical applications of skin-based biomechanical energy harvesting techniques and self-powered touch pad technology.

\section{EXPERIMENTAL SECTION}

Fabrication of the Skin-Based TENG and the Self-Powered Tactile Sensor System. The fabricated TENG is based on the contact/separation between a human skin patch, a PDMS film with a surface of micropyramid structures, and an ITO electrode, which was connected to a ground across an external load resistor. The micropyramid structures, with an average size of several micrometers, were fabricated by using an etched Si micropyramid template. For the self-powered tactile sensor system, a transparent and flexible ITO electrode array was fabricated using laser-cutting techniques. Then the fabricated PDMS film was overlaid on the ITO electrodes. Each ITO electrode was connected with a load resistor of $10 \mathrm{M} \Omega$, and the other end of the loading resistor was connected with the ground.

Measurement of the Fabricated Devices. In the current measurement process, the TENG was connected with a low-noise current preamplifier (Stanford Research SR570). The output voltage of the device with a loading resistance was measured by using a low-noise voltage preamplifier (>3 V, Keithley 6514 System electrometer; <3 V, Stanford Research SR560). A homemade data acquisition system was used to record the output voltage signals of the fabricated matrix in real time. The levels of wettability of the PDMS surface were characterized by the contact angle of water. The contact angles were measured by using a Rame-Hart goniometer that has a CCD camera equipped for image capture.

Conflict of Interest: The authors declare no competing financial interest.

Acknowledgment. This work was supported by U.S. Department of Energy, Office of Basic Energy Sciences (DE-FG0207ER46394), NSF, and the Knowledge Innovation Program of the Chinese Academy of Sciences (KJCX2-YW-M13).

Supporting Information Available: The additional figures include the cross-sectional SEM image of the fabricated PDMS film, the output voltage of the TENG without the micropyramid structures on the PDMS, the output current density of the TENG without the micropyramid structures on the PDMS, the enlarged output voltage of the device in Figure $4 c$, the detection limit of the pressure, and the output voltages of the device under different pressures. The additional movie files include the skin-based TENG for directly lighting up tens of green LEDs, the finger touch-induced biomechanical energy on a commercial cell phone, and the finger touch-induced biomechanical energy on a commercial keyboard. This material is available free of charge via the Internet at http://pubs.acs.org.

\section{REFERENCES AND NOTES}

1. Wang, Z. L.; Zhu, G.; Yang, Y.; Wang, S.; Pan, C. Progress in Nanogenerators for Portable Electronics. Mater. Today 2012, 15, 532-543.

2. Paradiso, J. A.; Starner, T. Energy Scavenging for Mobile and Wireless Electronics. IEEE Pervasive Comput. 2005, 4, 18-27.

3. Yang, Y.; Zhang, H.; Liu, Y.; Lin, Z.-H.; Lee, S.; Lin, Z.; Wong, C. P.; Wang, Z. L. Silicon-Based Hybrid Energy Cell for SelfPowered Electrodegradation and Personal Electronics. ACS Nano 2013, 7, 2808-2813.

4. Donelan, J. M.; Li, Q.; Naing, V.; Hoffer, J. A.; Weber, D. J.; Kuo, A. D. Biomechanical Energy Harvesting: Generating Electricity during Walking with Minimal User Effort. Science 2008, 319, 807-810.

5. Rome, L. C.; Flynn, L.; Goldman, E. M.; Yoo, T. D. Generating Electricity While Walking with Loads. Science 2005, 309, 1725-1728.

6. Mitcheson, P. D.; Miao, P.; Stark, B. H.; Yeatman, E. M.; Holmes, A. S.; Green, T. C. MEMS Electrostatic Micropower Generator for Low Frequency Operation. Sens. Actuators, A 2004, 115, 523-529.

7. Yang, R.; Qin, Y.; Li, C.; Zhu, G.; Wang, Z. L. Converting Biomechanical Energy into Electricity by a 
Muscle-Movement-Driven Nanogenerator. Nano Lett. 2009, 9, 1201-1205.

8. Fan, F.-R.; Tian, Z.-Q.; Wang, Z. L. Flexible Triboelectric Generator. Nano Energy 2012, 1, 328-334.

9. Guang, Z.; Pan, C.; Guo, W.; Chen, C.-Y.; Zhou, Y.; Yu, R.; Wang, Z. L. Tiboelectric-Generator-Driven Pulse Electrodeposition for Micropatterning. Nano Lett. 2012, 12, 49604965.

10. Wang, S.; Long, L.; Wang, Z. L. Nanoscale TriboelectricEffect-Enabled Energy Conversion for Sustainably Powering Portable Electronics. Nano Lett. 2012, 12, 6339-6346.

11. Lin, L.; Wang, S.; Xie, Y.; Jing, Q.; Niu, S.; Hu, Y.; Wang, Z. L. Segmentally Structured Disk Triboelectric Nanogenerator for Harvesting Rotational Mechanical Energy. Nano Lett. 2013, 13, 2916-2923.

12. Yang, Y.; Zhang, H.; Liu, R.; Wen, X.; Hou, T.-C.; Wang, Z. L. Fully Enclosed Triboelectric Nanogenerators for Applications in Water and Harsh Environments. Adv. Energy Mater. 2013, in press, DOI: 10.1002/aenm.201300376.

13. Hou, T.-C.; Yang, Y.; Zhang, H.; Chen, J.; Chen, L.-J.; Wang, Z. L. Triboelectric Nanogenerator Built inside Shoe Insole for Harvesting Walking Energy. Nano Energy 2013, in press, DOl: http://dx.doi.org/10.1016/j.nanoen.2013.03.001.

14. Bai, P.; Zhu, G.; Lin, Z.-H.; Jing, Q.; Chen, J.; Zhang, G.; Ma, J.; Wang, Z. L. Integrated Multilayered Triboelectric Nanogenerator for Harvesting Biomechanical Energy from Human Motions. ACS Nano 2013, 7, 3713-3719.

15. Shashoua, V. E. Static Electricity in Polymers. I. Theory and Measurement. J. Polym. Sci. 1958, 33, 65-85.

16. Maheshwari, V.; Saraf, R. F. High-Resolution Thin-Film Device to Sense Texture by Touch. Science 2006, 312, 1501-1504.

17. Wang C.; Hwang, D.; Yu, Z.; Takei, K.; Park, J.; Chen, T.; Ma, B.; Javey, A. User-Interactive Electronic Skin for Instantaneous Pressure Visualization. Nat. Mater. 2013, in press, DOI: 10.1038/NMAT3711.

18. Takei, K.; Takahashi, T.; Ho, J. C.; Ko, H.; Gillies, A. G.; Leu, P. W.; Fearing, R. S.; Javey, A. Nanowire Active-Matrix Circuitry for Low-Voltage Macroscale Artificial Skin. Nat. Mater. 2010, 9, 821-826.

19. Lipomi, D. J.; Vosgueritchian, M.; Tee, B. C.-K.; Hellstrom, S. L.; Lee, J. A.; Fox, C. H.; Bao, Z. Skin-Like Pressure and Strain Sensors Based on Transparent Elastic Films of Carbon Nanotubes. Nat. Nanotechnol. 2011, 6, 788-792.

20. Wu, W.; Wen, X.; Wang, Z. L. Taxel-Addressable Matrix of Vertical-Nanowire Piezotronic Transistors for Active and Adaptive Tactile Imaging. Science 2013, 340, 952-957.

21. Wang, Z. L.; Wu, W. Nanotechnology-Enabled Energy Harvesting for Self-Powered Micro/Nanosystems. Angew. Chem., Int. Ed. 2012, 51, 11700-11721.

22. Yang, Y.; Zhou, Y.; Wu, J. M.; Wang, Z. L. Single Micro/ Nanowire Pyroelectric Nanogenerators as Self-Powered Temperature Sensors. ACS Nano 2012, 6, 8456-8461.

23. Lin, Z.H.; Zhu, G.; Zhou, Y. S.; Yang, Y.; Bai, P.; Chen, J.; Wang, Z. L. A Self-Powered Triboelectric Nanosensor for Mercury Ion Detection. Angew. Chem., Int. Ed. 2013, 125, 51695173.

24. Lin, Z. H.; Xie, Y.; Yang, Y.; Wang, S.; Zhu, G.; Wang, Z. L. Enhanced Triboelectric Nanogenerators and Triboelectric Nanosensor Using Chemically Modified $\mathrm{TiO}_{2}$ Nanomaterials. ACS Nano 2013, 7, 4554-4560.

25. Yang, Y.; Lin, L.; Zhang, Y.; Jing, Q.; Hou, T.-C.; Wang, Z. L. Self-Powered Magnetic Sensor Based on a Triboelectric Nanogenerator. ACS Nano 2012, 6, 10378-10383.

26. Tuteja, A.; Choi, W.; Ma, M. L.; Mabry, J. M.; Mazzella, S. A.; Rutledge, G. C.; McKinley, G. H.; Cohen, R. E. Designing Superhydrophobic Surfaces. Science 2007, 318, 16181622.

27. Graz, I.; Kaltenbrunner, M.; Keplinger, C.; Schwödiauer, R.; Bauer, S.; Lacour, S. P.; Wagner, S. Flexible Ferroelectret Field-Effect Transistor for Large-Area Sensor Skins and Microphones. Appl. Phys. Lett. 2006, 89, 073501. 